Tohoku J. exp. Med., 1970, 102, 331-339

\title{
Recovery of the Kinetic and Tonic Components in M-wave after Repetitive Stimulation
}

\author{
Teruo Nakayama and Tetsuro Hori \\ Department of Physiology, Nagoya University School \\ of Medicine, Nagoya
}

\begin{abstract}
Nakayama, T. and Hori, T. Recovery of the Kinetic and Tonic Components in M-uave after Repetitive Stimulation. Tohoku J. exp. Med., 1970, 102 (4), 331 339. - 1) Electric stimuli were given to the ulnar nerve at the elbow in men and the responses (M-waves) were picked up by bipolar surface electrodes placed above the M. abductor digiti V. 2) The kinetic component of the M-wave has a lower threshold than the tonic component in the strength-duration curve. 3 ) With paired stimulation, both the kinetic and tonic components were noticed on the second response even at a stimulus interval of 2 msec. 4) Similar responses were obtained successively by the repetitive stimulation of less than 20 per sec. At the rate of 30 per sec, the tonic activity was abolished in the fourth or fifth and in the subsequent responses. 5) A test stimulus was applied 10 msec after 10 conditioning shocks. When the rate of conditioning stimulus was more than 10 per sec, the tonic component was abolished and the 'pure' kinetic responses were obtained. Full recovery of the tonic component requires 200 and $300 \mathrm{msec}$ after 10 impulses at the rate of 10 and 50 per sec, respectively. The inhibition of kinetic component was observed after conditioning at 200 per sec and the full recovery occurred within $100 \mathrm{msec}$. 6) It is suggested that during repetitive stimulation the transmitter available for immediate release is exhausted faster in the tonic motor nerve terminals than in the kinetic nerve terminals, probably due to a slower mobilization or larger quantal liberations of transmitter.

repetitive nerve stimulation; kinetic and tonic motoneurons; transmitter release
\end{abstract}

Spinal $\alpha$-motoneurons are divided functionally into two groups, i.e., the kinetic and tonic motoneurons and their responses to antidromic and orthodromic stimulations have been studied in cats by Eccles et al. ${ }^{1}$ and Granit et al. ${ }^{2}$ The tonic motoneurons, which receive a more dense projection of the Group Ia afferents, are subjected to the post-tetanic potentiation and the duration of after-hyperpolarization is longer than in the kinetic motoneurons.

Muscle action potentials in response to the percutaneous stimulation of the nerve trunk in man are often called $\mathrm{M}$ - and $\mathrm{H}$-waves. The former is evokad directly by stimulation of the motor nerve, while the latter is induced reflexly by stimulation of the proprioceptive sensory afferents. In our previous studies, ${ }^{3,4}$ it was found that these waves are composed of two components, i.e., the kinetic and tonic activities. The conduction velocity of the kinetic motor fiber in man is faster than that of the tonic fiber, so that the vector summation of these two kinds of

Received for publication, July 25, 1970. 
muscle action potentials results in a complex wave form. As the upward deflection of the kinetic potential is usually canceled by the downward deflection of the tonic potential, the peak-to-peak amplitude of the compound muscle action potential is reduced in comparison with the pure kinetic and pure tonic action potentials. This explains why the amplitude of $\mathrm{M}$ - and $\mathrm{H}$-waves recorded by surface electrodes is not always proportional to the mechanical response of the muscle.

In this paper, $\mathrm{M}$-waves in man were studied during and after repetitive stimulation of the motor nerve in reference to the kinetic and tonic activities.

\section{Methods}

Subjects were staffs at the Department aged 20 to 40 years. Isolated square pulses were delivered through disk electrodes placed above the ulnar nerve at the elbow. The stimulus intensity and the duration were variable, ranging from 25 to $60 \mathrm{~V}$ and 0.1 to 3.0 msec, respectively. A consistent maximum response was obtained in most cases with the stimulus of about $40 \mathrm{~V}$ and $1 \mathrm{msec}$. Muscle action potentials were recorded from the M. abductor digiti $V$. Two surface electrodes of $2 \times 2 \mathrm{~mm}$ silver plate were placed on the skin above the muscle and a large bandage-like electrode was fixed at the middle part of forearm for an electric grounding. The mode of stimulation in each experiment will be mentioned in the Results.

\section{RESULTS}

Strength-duration curve. The duration of the stimulus was varied from 5 to 0.1 msec, and at each pulse duration the stimulus voltage was increased to an intensity which gives rise to a noticeable muscle action potential. The threshold thus obtained is for the kinetic component. With a further increase in stimulus intensity, the wave form of the muscle action potential undergoes a change showing a notch or hump on it, which indicates a participation of the tonic activity. One of the results obtained was plotted in Fig. 1 as the strength-duration curve. In all of 6 subjects and at all pulse durations tested, the tonic response showed a higher threshold.

Paired stimulation. Two successive stimuli were given to the ulnar nerve and the two responses of the abductor digiti $\mathrm{V}$ muscle were compared to see if either kinetic or tonic component is suppressed in the second response, as the stimulus interval becomes shorter. When the stimulus interval is short, the second response occurs before the first response comes to an end. To obtain a clear form of the second response, therefore, fifty responses to paired stimuli were added on the data processing computer (Nihonkohden ATAC-401), from which fifty responses to a single stimulus were subtracted. Records of the second response thus obtained, as shown in Fig. 2, are not much different from the first responses at the stimulus interval of $4 \mathrm{msec}$ or longer. At an interval of $2 \mathrm{msec}$, the amplitude of the second response is reduced but the notch is seen, indicating that both the kinetic and tonic activity are still involved. Thus, a paired stimulation failed to separate the two components. 


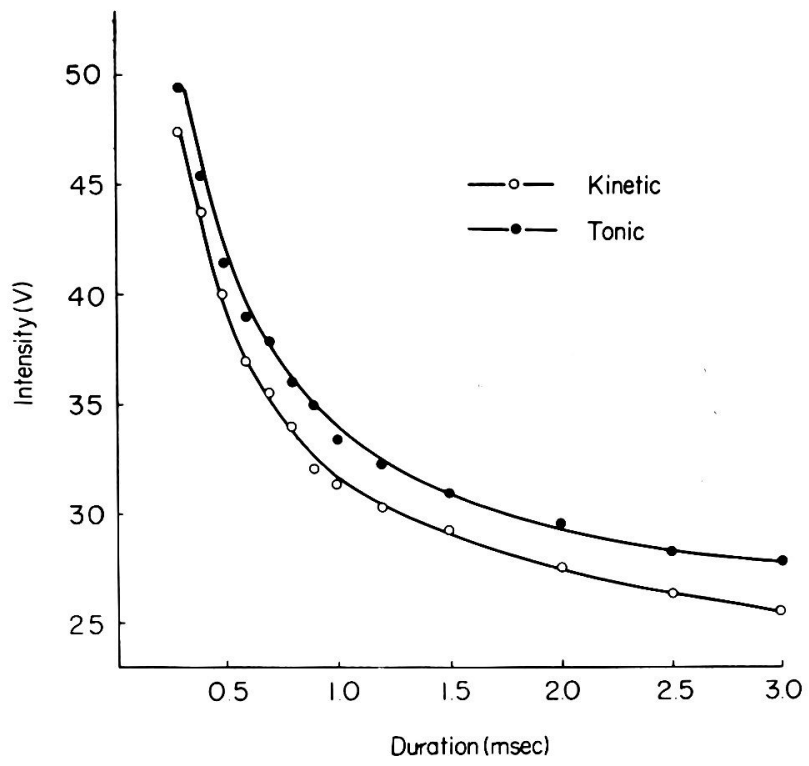

Fig. 1. Strength-duration curve. Electric stimuli were delivered to the ulnar nerve at the elbow and the threshold of the kinetic and tonic components was measured on the evoked electromyogram of the abductor digiti Vmuscle.
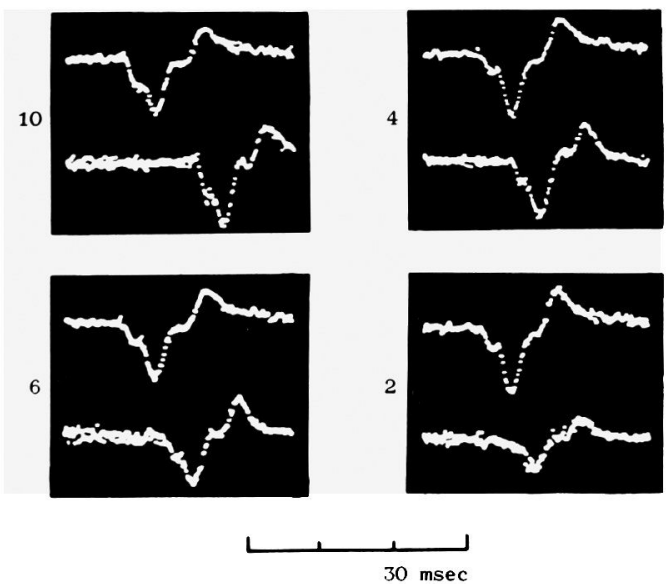

Fig. 2. Average of fifty M-waves in response to paired stimuli. In each record, the upper and lower traces are the first and the second responses. In the lower traces, the first responses were abolished by reduction. Numbers indicate stimulus intervals in msec.

As is well known in a curarized nerve-muscle preparation, paired stimuli given at the interval of a few msec give rise to the increased amplitude of the second endplate potential. ${ }^{5}$ This facilitation of the neuromuscular transmission is likely to occur in the present studies and might explain the increased amplitude of the second response at the stimulus interval of $10 \mathrm{msec}$, as is seen in Fig. 2. 
Repetitive stimulation. The ulnar nerve was stimulated repetitively at the rate of $1,5,10,20$ and 30 per second. As is shown in Fig. 3, the wave form did not change at the stimulation rate of 20 per sec. In this response, the initial downward deflection is followed by the second downward deflection. The latter is considered to be due to the tonic activity. At the rate of 30 per second, the second deflection is less and less distinct in the second and the third responses. After the fifth response, the second deflection is no longer observed and the subsequent stimulation produced fairly the same responses which are considered to be purely kinetic. The results clearly indicate that the tonic activity fails to respond at frequencies above 30 per second. In good agreement with this, it has been long known that the maximum discharge rates during sustained voluntary contractions are approximately 20 and 50 per second in the tonic and kinetic neuromuscular units, respectively. ${ }^{6}$

20

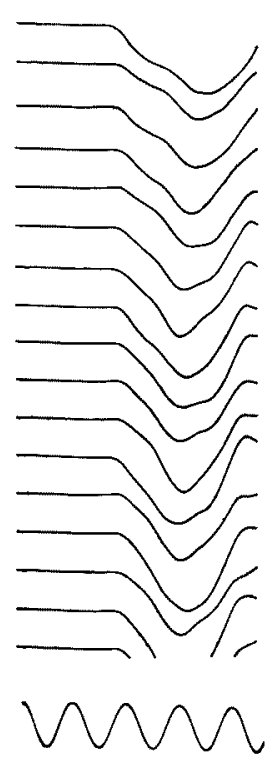

500
30

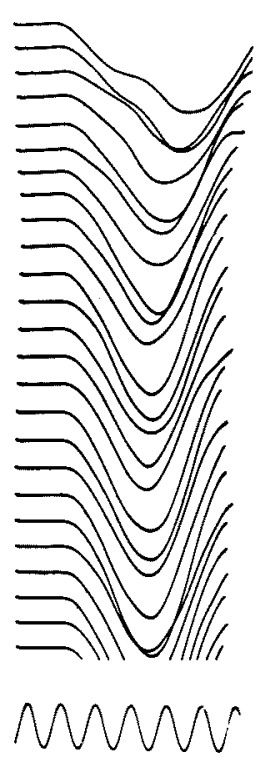

$1000 \mathrm{cps}$

Fig. 3. M-waves in repetitive stimulation at the rate of 20 and 30 per sec.

Recovery of $M$-wave after repetitive stimulation. A test stimulus was given at 10 to 300 msec after a train of 10 successive nerve volleys at the rate of $5,10,25$, 50,100 and 200 per sec. After conditioning at 5 per sec, the response to the test stimulus given $10 \mathrm{msec}$ later was the same as control (Fig. 4). The recovery of the response tock 100 to 150 msec after the repetitive stimulation of 10 per sec. When the conditioning was 25 per sec, the tonic component disappeared in the responses taken at 50 and $100 \mathrm{msec}$ interval. As is shown in the left column of Fig. 4, the response shows a 'pure' kinetic component, free from contamination of the tonic 

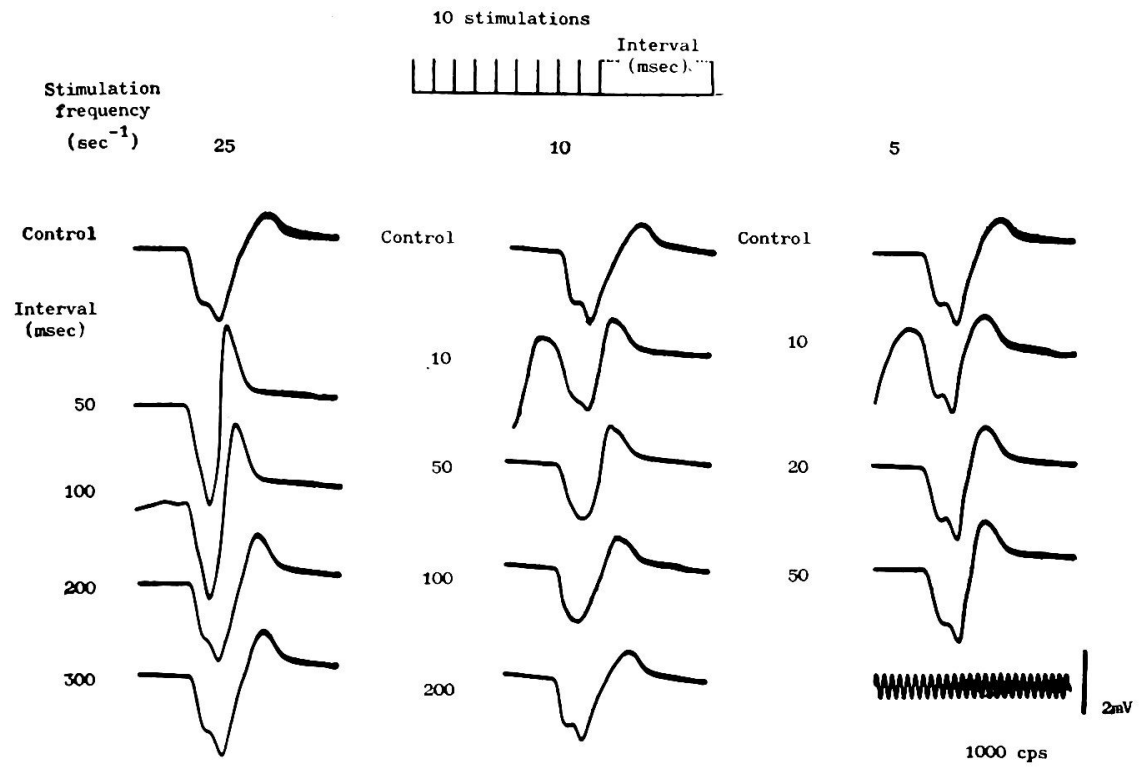

Fig. 4. Recovery of M-wave. Test stimulus was given after 10 repetitive stimulation at 25,10 and 5 per sec.

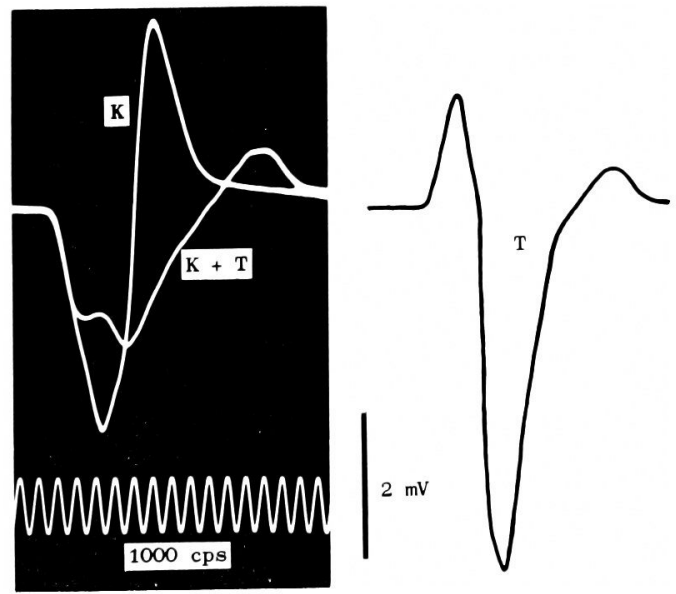

Fig. 5. Analysis of $M$-wave. ' $K+T$ ' is a control response. ' $K$ ' was obtained after repetitive stimulation at $\mathbf{5 0}$ per sec. A vector difference between ' $\mathrm{K}+\mathrm{T}$ ' and ' $\mathrm{K}$ ' is presented as ' $\mathrm{T}$ '.

component. The full recovery of the tonic activity was obtained $200 \mathrm{msec}$ after the train stimulation. The control response and the 'pure' kinetic response were superimposed in Fig. 5. The vector difference of these two waves was presented in the right half of the figure as the tonic component, the triphasic deflections of which indicate that the wave is a 'pure' response of the tonic activity. The 
$10200 /$ sec-stimulations
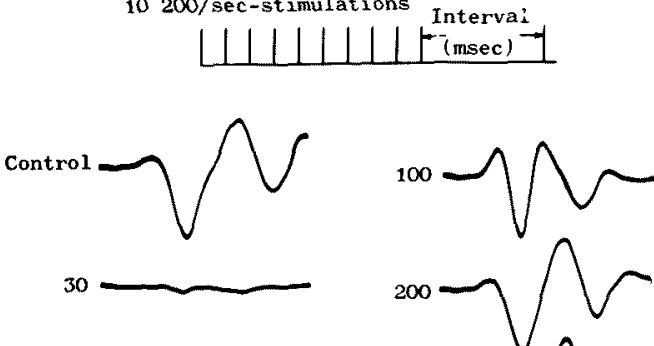

40
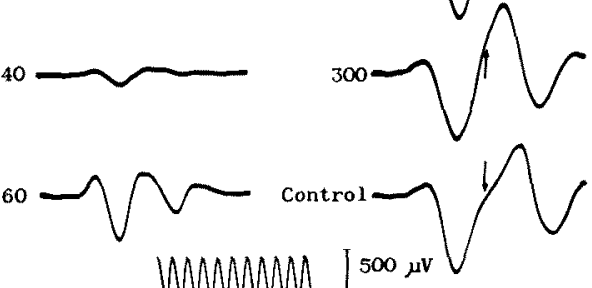

WMAMA $[500 \mu \mathrm{v}$

1000 cps

Fig. 6. Recovery of M-wave. Test stimuli were given at various intervals after 10 repetitive stimulation at 200 per sec. Arrows indicate the hump caused by the tonic activity.

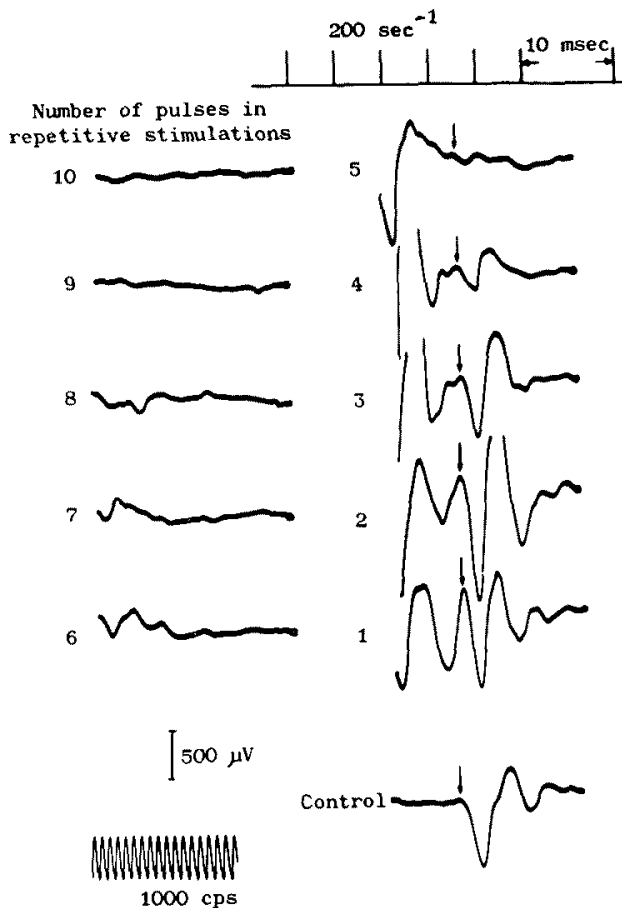

Fig. 7. Recovery of $M$-wave. Test stimuli were given $10 \mathrm{msec}$ after the repetitive stimulation at 200 per sec. Number of repetitive stimulation was reduced from 10 to 1 . Arrows indicate the time of onset of $M$-waves in response to test stimuli. 
results obtained after stimulation at 50 per sec was similar to those at 25 per sec. This means that once the tonic activity is inhibited by repetitive stimulation, a further increase in stimulus frequency does not produce a different response until the frequency is increased enough to inhibit the kinetic activity. The kinetic component was still activated by the test stimulus given $10 \mathrm{msec}$ after conditioning at 100 per sec. When the rate of repetitive stimulation was increased to 200 per sec, the response was completely depressed for about a period of $30 \mathrm{msec}$, as is shown in Fig. 6. The recovery of the kinetic component began at $40 \mathrm{msec}$ and completed in $100 \mathrm{msec}$ after the repetitive stimulation, whereas the depression of the tonic component lasted longer up to $300 \mathrm{msec}$.

In Fig. 7, repetitive stimulation was applied at the rate of 200 per sec and the number of pulses was reduced to see how many pulses are necessary to inhibit the kinetic activity. The test stimulus was given $10 \mathrm{msec}$ after the last pulse of the conditioning. The appearance of the kinetic component was noticed when the number of stimulating pulse was decreased to four, while the tonic component was barely detectable only after one conditioning impulse.

\section{Discussion}

The present studies reconfirmed the fact that the electromyogram recorded by surface electrodes is a vector summation of two action potentials caused by kinetic and tonic muscle fibers. Differences in characteristics have been reported on various aspects of these two activities. ${ }^{1-4,7}$ In physiological conditions, the upper limit of the firing rate of spinal motoneurons is limited by the recovery of the membrane potential. The duration of after-hyperpolarization of the kinetic motoneuron is 50 to $110 \mathrm{msec}$, while that of tonic motoneuron is 130 to 240 msec. ${ }^{1}$ In accordance with this, the maximum firing rate of the tonic motor unit is lower than that of the kinetic motor unit. Our previous paper revealed that in comparison with the tonic component, the kinetic component shows a higher threshold to the dorsal root stimulation and a lower threshold to the ventral root stimulation. $^{3}$ The conduction velocity of the kinetic motor fiber was found faster than that of tonic fiber in cat' as well as in man. ${ }^{4}$ The contraction curve of the tonic muscle fiber has been shown to be slowly rising and falling. ${ }^{1}$ Thus, the available evidence indicates that both the nerve fiber and muscle fiber are slower in action in the tonic activity. It is not possible to draw from the results presented in this paper a precise recovery curve for the kinetic and tonic activities. Some recovery characteristics, however, were clarified on the two activities. Repetitive stimulation at the rate of 25 per sec abolished the tonic activity, while the rate of stimulation had to be increased up to 200 per sec to inhibit the kinetic activity. Once inhibited by repetitive stimulation, a full recovery required $100 \mathrm{msec}$ for the kinetic and $300 \mathrm{msec}$ for the tonic component. When paired stimuli were delivered, the second response was identical with a control response even at the stimulus interval of $4 \mathrm{msec}$. 
These differences in responses between the kinetic and tonic activities are largely attributable to the neuromuscular transmission rather than to the nervous conduction. Potentiation and depression of the endplate potential observed after conditioning shock or during short tetanus is of presynaptic origin, presumably due to an increased and decreased number of acetylcholine quanta released from nerve terminals. ${ }^{5,8,9}$ During repetitive stimulation, the amplitude of the 'pure' kinetic responses was greatly increased as shown in Figs. 3 and 4 . This increase may be simply explained as a result of inhibition of the tonic action potential, but a possible potentiation of the kinetic activity cannot be ruled out. It has been generally suggested that only a small fraction of total acetylcholine content exists in the synaptic vesicles immediately adjacent to the terminal membrane ${ }^{10,11}$ and 33 to $45 \%$ of this available transmitter is released by one impulse under normal condition. In addition to the discharge of some vesicles, the presynaptic impulse is proposed as causing other vesicles to transfer into the zone fronting the synaptic cleft. ${ }^{10}$ This mobilizing action of the impulse, occurring independently of its releasing function, would be speeded up during tetanic stimulation. ${ }^{12}$ Thus the rate of repetitive stimulation to which the kinetic and tonic components can follow depends on the amount of the available transmitter at any one instant, which would be determined by the rate of two opposing processes, i.e., depletion by release and mobilization. The present studies suggest that during the repetitive stimulation the transmitter available for release is exhausted at a lower rate of stimulation in the tonic motor nerve terminals than in the kinetic nerve terminals. This could be caused either by a larger quantal liberation per single nerve volley, or slower mobilization processes in the tonic nerve terminals. But at present no information is available on the postulated differences in the acetylcholine kinetics at neuronal terminals between tonic and kinetic motor fibers.

\section{References}

1) Eccles, J.C., Eccles, R.M. \& Lundberg, A. The action potentials of the alpha motoneurones supplying fast and slow muscles. J. Physiol. (Lond.), 1958, 142, 275-291.

2) Granit, R., Henatsch, H.D. \& Steg, G. Tonic and phasic ventral horn cells differentiated by post-tetanic potentiation in cat extensors. Acta physiol. scand., $1956,37,114-126$.

3) Nakayama, T. \& Hori, T. Tonic and kinetic components of the evoked electromyogram. Jap. J. Physiol., 1967, 17, 415-428.

4) Nakayama, T. \& Hori, T. Conduction velocity of the tonic motor fibers in man. Tohoku J. exp. Med., 1967, 93, 391-394.

5) del Castillo, J. \& Katz, B. Statistical factors involved in neuromuscular facilitation and depression. J. Physiol. (Lond.), 1954, 124, 574-585.

6) Adrian, E.D. \& Bronk, D.W. The discharge of impulses in motor nerve fibres. Part II. The frequency of discharge in reflex and voluntary contractions. $J$. Physiol. (Lond.), 1929, 67, 119-151.

7) Nakayama, T. \& Hori, T. Reticular and cortical influences on the kinetic and tonic components of evoked electromyogram. Tohoku $J$. exp. Med., 1967, 93, 395-403.

8) Liley, A.W. The quantal components of the mammalian end-plate potential. $J$. Physiol. (Lond.), 1956, 133, 571-587. 
9) Otsuka, M., Endo, M. \& Nonomura, Y. Presynaptic nature of neuromuscular depression. Jap. J. Physiol., 1962, 12, 573-584.

10) Eccles, J.C. The Physiology of Synapses. Springer-Verlag, Berlin. 1964, pp. 92-93.

11) Liley, A.W. \& North, K.A.K. An electrical investigation of effects of repetitive stimulation on mammalián neuromuscular junction. $J$. Neurophysiol, $1953,16,509-$ 527.

12) Hubbard, J.I. Repetitive stimulation at the mammalian neuromuscular junction, and the mobilization of transmitter. $J$. Physiol. (Lond.), 1963, 169, 641-662. 